\title{
Competition and cooperation in a bidding model of electrical energy trade
}

\author{
Dávid Csercsik
}

The final publication is available at Springer via http://dx.doi.org/10.1007/s11067-015-9310-

\begin{abstract}
A cooperative game-theoretic framework is introduced to study the behavior of cooperating and competing electrical-energy providers in the wholesale market considering price-preference rational consumers. We study the physical and economic aspects of the power transmission system operation focussing on the incentives for group formation. We analyze the interactions of generators in an idealized environment described by a DC load flow model where the network is lossless and is operated by an independent network operator who ensures network stability and fulfillment of consumption needs while taking into account the preferences of consumers over generators. We show that cooperation of generators may be necessary to divert consumers from their previous providers. In the second part of the paper we assume an iterative process in which the generators publish their price offers simultaneously, based on which the consumers preferences are determined. We study the dynamics of the prices and profits as the system evolves in time while each coalition is trying to maximize its expected profit in each step. The model deals with network congestion and $n-1$ line-contingency reliability as not every generator-consumer matching is allowed to ensure the safe operation of the transmission system. The profit of the generators is determined as the dif-
\end{abstract}

This work was supported by the Hungarian Academy of Sciences under its Momentum Programme (LP-004/2010), by the Hungarian National Fund (OTKA NF-104706) and by the Fund KAP-1.2-14/001.

Dávid Csercsik

Pázmány Péter Catholic University, Práter U. 50/A 1083 Budapest, Hungary

Tel.: +36-1 8864700

Fax: +36-1 8864724

Game Theory Research Group

Centre for Economic and Regional Studies of the Hungarian Academy of Sciences, Budaörsi

U. 45, 1112 Budapest, Hungary

Tel.: +36-1 3092652

Fax: +36-1 3193136

E-mail: csercsik@itk.ppke.hu 
ference between their income and their production cost, which is a quadratic concave function of the production amount.

Any non-monopolistic proper subset of the generators may cooperate and harmonize their offered prices to increase their resulting profit. Since we allow the redistribution of profits among cooperating generators, a transferableutility game-theoretic framework is used. Furthermore, as cooperation affects the outsiders as well, the resulting game is defined in partition function form. The model is able to demonstrate some interesting benefits of cooperation as well as the effect of market regulations and asymmetric information on the resulting profits and total social cost.

Keywords Networks · Power Transmission · Game theory · Externalities

\section{Introduction}

Because of its extreme importance, power system economics (Kirschen and Strbac, 2004) has been an intensively researched interdisciplinary area. The trends of electricity market liberalization, together with occasionally rapidly extending consumption in the long term and consumption peaks in the short term, put increasing load on system operators and authorities responsible for network operation and expansion.

If one wishes to analyze the electrical-energy market as interactions of market participants, one has to take into account that the possible interactions are constrained by laws of physics as well as by market regulations.

Studies approaching the topic from the engineering discipline dominantly consider optimal power flow (Wood and Wollenberg, 2012; Conejo and Aguado, 1998) and direct current optimal power flow (DCOPF) problems. In these approaches the aim is to minimize the total cost of system operation under various constraints. These papers, among others, study how the topology of the network affects transmission efficiency and thus how the usage of flexible ac transmission systems (FACTS) (Hingorani, 1993; Hingorani et al, 2000; Song and Johns, 1999) may be optimized. Unit commitment (Sheble and Fahd, 1994) addresses the problem of economically optimizing generator schedules over a short-term horizon subject to demand and other constraints. The unitcommitment problem has been approached by various optimization methods (see e.g. (Cheng et al, 2000; Zhuang and Galiana, 1990)), and reformulated to include transmission constraints (Tseng et al, 1999).

Regarding safety issues of power system operation, optimal transmissionswitching models (Fisher et al, 2008; Hedman et al, 2008; ONeill et al, 2010), which formulate the optimization as a mixed-integer problem, usually assume $n-1$ contingency reliability (Hedman et al, 2009), which means that the effects of single line and generator failures on the network are included in the analysis. The latest models of Hedman et al (2010) even include generator startup and shutdown costs as well. 
While the above papers provide valuable insight in the problems of optimal network design and operation, they do not assume profit-oriented generators and neglect several economical incentives of the market participants.

When studying the economic aspects of electric-power transmission networks, most of the research has focussed on the topics of competition, market power and regulation (Gilbert et al, 2004; Neuhoff et al, 2005; Chen et al, 2006). A large-scale spatial optimization model of the European market considering nodal or zonal pricing is presented in (Leuthold et al, 2012). The proposed model is used for the prediction of optimal congestion management, expansion planning and generator investments under network constraints. A generalized Nash equilibrium model of market coupling in the European market is presented in (Oggioni et al, 2012). The Cournot assumption has been used in several papers (Cardell et al, 1997; Yao et al, 2004; Sauma and Oren, 2007) to analyze the market power in electrical energy trade models. The paper (Metzler et al, 2003) focuses on the effects of arbitrage in a Nash-Cournot equilibrium model. A further example of Nash-Cournot equilibrium models applied to power markets may be found in the paper (Gabriel et al, 2013) The paper of Ruiz et al (2012) assumes profit maximizing providers, network constraints and uses an equilibrium framework to predict prices and profits in a pool-based electricity market. Pool-based markets are detailed furthermore in (De la Torre et al, 2003, 2004) These and similar models (Bakirtzis et al, 2007) usually assume elastic demand and piecewise constant price-demand curves and market clearing, and lead to MPECs (mathematical program with equilibrium constraints) or EPECs (equilibrium problems with equilibrium constraints) (Ehrenmann, 2004).

The articles (Hobbs and Kelly, 1992; Bai et al, 1997) already use game theory for transmission analysis. Hobbs and Kelly (1992) use static cooperative models to calculate the possible outcomes of short-run transmission games and noncooperative Stackelberg games to model long-run games in which the amount of transmission capacity is a decision variable. The paper by Bai et al (1997) describes an open-access transmission method for maximizing profits in a power system, where transmission losses are considered. The proposed method is based on the Nash bargaining game for power-flow analysis in which each transaction and its optimal price is determined to optimize the interests of individual parties.

The paper of Orths et al (2001) describes a game-theoretic approach of a multi-criteria optimization problem related to transmission planning and operation. A strategic-gaming approach is described in (Kleindorfer et al, 2001). The paper of Harrington et al (2005) describes a collusive framework motivated by power-generation auctions in which players coordinate in order for each to gain higher payoffs than those determined by the Nash equilibrium solution. This model has been extended by Liu and Hobbs (2013) to take transmission constraints into account as well.

Gately (1974) was probably the first to apply cooperative game theory to planning investments of electrical-power systems. In this paper the concepts of the core and the Shapley value are used to determine the mutually accept- 
able set of final payments. The paper (Evans et al, 2003) describes a cost assignment model for electrical transmission system expansion is using kernel theory. The study by Sauma and Oren (2007) addresses network expansion as well and shows that in the presence of imperfect markets the elimination of network congestion may not necessarily improve social welfare. The methods of cooperative game theory have been applied for the analysis of the transmission expansion problem both in the case of centralized and decentralized environment (Contreras, 1997; Contreras and Wu, 1999, 2000; Contreras et al, 2009).

Corresponding to the pricing problem of electricity markets, Bolle (1992) compares constant and spot prices in oligopolistic models, using supply functions. Fuller (2005) analyzes the relationship between the hourly spot price at a node, and the prices at all nodes adjacent to it. Considering market regulations, Singh et al (1998) compare a nodal pricing framework with cost-allocation procedures in the case of competitive electricity markets, and analyze some game-theoretic aspects of the proposed model. The paper of Ding and Fuller (2005) considers nodal, zonal and uniform marginal prices and emphasizes that the nodal marginal price correctly accounts for transmission constraints and losses in some cases.

As pointed out by Liu and Hobbs (2013) transmission constraints can be exploited by strategic firms to enhance their market power, and collusive generators can strategically exploit transmission congestion and reap additional profits compared to the situation without congestion. Our aim in this paper is to introduce a transferable-utility game-theoretic model (Arnold and Schwalbe, 2002 ), which is able to describe the various levels of generator firms' cooperation, while taking into account profit-motivated generators, various market regulations and engineering type modelling assumptions of the transmission system as well ( $n-1$ line contingency reliability). It is natural to assume that under certain market regulations, coalition formation of generators may be illegal; however coalitions may be interpreted also as generators belonging to the same energy-providing company.

The proposed market model is liberalized in the sense that consumers, who are characterized by zero elasticity demand, may choose their preference over power providers who compete for them based on their price; but each consumer is be assigned to only one provider at a time. We assume that the formation of coalitions who could raise their prices as high as they wish is prohibited.

To analyze how the prices and profits evolve in time, we study an iterative process in which the generators publish their price offers simultaneously in each step, based on which the consumers preferences are determined. Each coalition tries to optimize its expected profit in each step, based on the price offers of the previous step. Our aim in this paper is not to study the equilibrium properties of the proposed framework, but to get an impression of how the dynamics of prices and profits are affected by coalition formation in various cases.

A further aim is to analyze the effect of market regulations (allowance or prohibition of zonal pricing) and asymmetric information on the resulting profits of the generators and on the total social cost (the total amount of 
money the consumers pay to the generators in order to supply their needs). The approach of cooperative game theory has the capability to describe various levels of cooperation between oligopolistic and, in this case, two coalitioncompetitive scenarios. In transferable-utility cooperative games the concept of superadditivity is used for the description of whether a coalitional merger brings benefits to the merging coalitions, and whether this benefit depends on the behavior of other players (not involved in the merger) or not. A such analysis may provide a valuable tool for the identification of incentives for cooperation - in other words it may predict which generators may be motivated for cartel formation.

\section{Materials and Methods}

\subsection{Game-theoretic preliminaries}

Let $N=\{1,2, \ldots, n\}$ be the set of players, and its non-empty subsets are the coalitions, denoted by $C$. A partition $P$ is a set of disjoint coalitions; $P=$ $\left\{C^{1}, C^{2}, \ldots, C^{m}\right\}$, where their union is $N$, i.e., players in set $K$ cooperate if and only if $K \in P$. The set of partitions is $\mathcal{P}$ and the set of partitions of $C \subseteq N$ is $\mathcal{P}(\mathcal{C})$. A partition function; $V: \mathcal{P} \rightarrow\left(2^{N} \rightarrow \mathbb{R}\right)$ assigns a characteristic function $(v)$ to each partition. A characteristic function $v: 2^{N} \rightarrow \mathbb{R}$ assigns to each coalition $C \subseteq N$ its worth or payoff $v(C)$, with the convention that $v(\emptyset)=0$. A cooperative game with transferable utility in partition function form, or briefly PFF-game (Thrall and Lucas, 1963), is a pair $(N, V)$, where the worth of a coalition may be different in each partition.

Externalities describe the effect of the formation of a coalition on third parties (e.g. if A and B forms a coalition, they affect the payoff of $\mathrm{C}$ as well). Externalities may be positive if the payoff of a third-party coalition increases when a particular coalition forms or negative if the payoff decreases.

\subsection{Modelling assumptions}

We consider a model of the electricity market and the underlying energytransmission network. For the description of the underlying network we will use the DC load flow model and the corresponding terminology defined in (Csercsik and Kóczy, 2011) ${ }^{1}$. The DC load flow model has been widely used

\footnotetext{
1 The most important feature of a DC load flow model is that if we make some key assumptions regarding the power grid (neglect the real part of line impedance, and assume the same peak voltage at every node) then the mathematical form of the equations describing the real power flow will be equivalent to the equations describing the flow of the DC current in a resistance network. The admittance values $(Y)$ and the injected/consumed energy amounts of the nodes uniquely determine the energy flows on the edges (branches or lines) of the network, which can be obtained by the solution of a system of linear equations. In addition to its admittance value, each edge is characterized by a transfer capacity $(\bar{q})$, which corresponds to the maximal amount of energy which can be transferred on it. Furthermore, as a most simple approach we neglect transmission losses.
} 
among papers analyzing power-system economics (see e.g. (Tseng et al, 1999; Yao et al, 2004; Sauma and Oren, 2007)).

The DC load flow model is based on the graph of the transmission network. For the sake of simplicity we will assume that every node of the energytransmission network is assigned to a single generator or consumer. The most straightforward interpretation of the model is that we study the high-voltage energy-transportation networks, in which case consumers correspond to local energy providers who own mid-voltage networks.

In the following we summarize the assumptions regarding our model

- We assume $n_{g}$ generators, which are considered as the players of the game, $n_{c}$ consumers and $n_{l}$ lines.

- Any consumer is allowed to buy energy from only one generator.

- As the focus of this study is to analyze the competition and cooperation of energy providers under various market regulations (prohibition or allowance of zonal pricing), we neglect the demand elasticity of consumers (it is supposed that consumers buy their total required amount of energy, even if not on the most preferred price), and assume perfectly predictable loads. This might be a relevant assumption if we consider that the consumers are local-area suppliers who have to fulfill residential consumer demands and can not propagate the production-cost changes in the short term (eg. they have long-term contracts with the residential consumers and buy energy with-short term contracts. For more on demand elasticity in energy markets see (Bernstein et al, 2006; Berndt and Wood, 1975)).

- Depending on the actual market regulation, each generator may offer nodal (consumer-dependent) or universal prices (same for all consumer), which determine the priority of consumers over the generators.

- Each generator has limited production capacity, which may limit the number of consumers it can supply with energy.

- Generators act as the players of the game, while rational price-priority consumers are assumed.

- Generator $j$ offers energy for sale to consumer $k$ at price $p o_{k}^{j}$. If no zonal pricing is allowed $p o_{k}^{j}=p o^{j} \forall k$. We summarize the offers in the price-offer matrix $P O$, where $[P O]_{k, j}=p o_{k}^{j}$.

Considering the prices offered by the generators, the consumers (who are assumed to be price-preference rational ${ }^{2}$ ) set up preferences describing from which generator they want to purchase their required amount of energy. The preference of each consumer can be regarded as an ordering of the generators. Since the prices offered by the providers may be the same, the preference ranking is not necessary strictly monotonic.

Based on these preferences and network characteristics, the independent system operator (ISO) determines a matching $\mu$ of generators and con-

\footnotetext{
2 Which means they set up preferences based only upon the price offers. They prefer the generator most who offers them energy at the lowest price, the second most preferred is the one offering the second lowest price, etc.
} 
sumers. A matching $\mu$ is a set of generator-consumer pairs, in which every consumer is included in one and only one pair.

Definition 1 We call a matching $\mu$ between generators and consumers feasible is every generator's capacity allows it to fulfill of all those consumers's requirements who are assigned to it.

Definition 2 We call a matching $\mu$ stable if the resulting network configuration implies a stable state of the network. A stable state of the network is when no line is overloaded, and no instantaneous failure of any line may lead to overload of any other line in the network (n-1 line contingency in other words).

First the ISO identifies if the most preferred matching (the matching which implies the lowest resulting total cost for the consumers) is feasible and stable. In case it is, it will be the resulting matching. If the most preferred matching is not stable or not feasible, the ISO analyzes all matchings where the preferences are violated in one case (one consumer is assigned to its second most preferred generator), and from the stable and feasible matchings it chooses the one which brings the least additional cost to the consumer who is subject of preference violation (who is not assigned to its most preferred generator). If multiple matchings exist in which the additional cost is nearly equal, the one with higher stability margin will be realized. The stability margin for a matching $\mu$ may be defined as follows. If the matching is unstable, the stability margin is 0 . If the matching is stable, the value of the stability margin $\mathcal{S}$ can be defined as

$$
\mathcal{S}(\mu)=\sum_{i} \sum_{j \neq i} \bar{q}_{j}-q_{j}^{i}
$$

where $q_{j}^{i}$ is the flow on line $j$ in the case of the failure of line $i$, and $\bar{q}_{j}$ is the maximal allowed flow on line $j$.

If no such matching exists, the additional costs of the matchings with two preference violations will be analyzed, etc. If there is at least one feasible and stable configuration, the algorithm will stop. ${ }^{3}$

This matching will determine the resulting prices. $p_{k}^{j}$ denotes the price which is paid by consumer $k$ to generator $j$ for one unit of energy. $p_{k}^{j}=p o_{k}^{j}$ for all $\{k, j\}$ pairs in the matching.

3 A possible alternative for this matching method is when the ISO calculates all possible matchings, and chooses that stable and feasible one, which implies the lowest total cost for the consumers. As the number of all possible matchings grows exponentially with the number of generators and consumers, this would significantly increase the computational demand of the algorithm (see the details in Fig. 1). The application of the preference-violation based iterative search, which provides the social optimum (the lowest total social cost) in most cases, may be regarded as a trade-off, which significantly lowers the computing capacity needed for the ISO and thus enhances scalability, but theoretically may lead to suboptimal solutions. 
In other words, the ISO ensures the fulfillment of the matching most preferred by the consumers under the constraints implied by the network stability and feasibility requirements. The parameters of this configuration depend on the actual prices offered by the generators.

- The generators are fully aware of the ISO's matching algorithm.

- The concept of plant utilization is important when analysing the costs of generating electricity. It can be observed that a plant with low utilization inevitably has a high unit cost of production because the same investment and fixed costs of operation and maintenance are recovered over fewer units of production. As the most simple approach, we assume that generation cost per unit is linear decreasing function of generated quantity ${ }^{4} c^{j}=$ $a^{j}-m^{j} Q^{j}$ where $a^{j}[\$]$ and $m^{j}>0[\$ / \mathrm{MWh}]$ are the constants describing the production characteristics of generator $j$ (which depend on the applied technology), while $Q^{j}[\mathrm{MWh}]$ is the total energy quantity produced by the generator. The total generation cost of a generator can be formulated as: $C^{j}=c^{j} Q^{j}$

- The income of a certain generator is the sum of the incomes from various consumers:

$$
I^{j}=\sum_{k \in S^{j}} p_{k}^{j} Q_{k}
$$

where $S^{j}$ is the set of consumers who buy energy from generator $j . Q_{k}$ [MWh] denotes the quantity bought by consumer $k$.

- Profit of player $j$ denoted by $\Phi^{j}[\$]$ can be calculated as the difference of income and generation cost for the player: $\Phi^{j}(P O)=I^{j}(P O)-C^{j}\left(Q^{j}\right)$. In the case of full or pure competition (all singleton coalitions) it is assumed that all players try to maximize their own expected profit ${ }^{5}$.

- One straightforward interpretation of coalitions is to assume that the generators of a certain coalition belong to the same company (we would like to emphasize that still in this case we assume that consumers buy energy from generators, not from the coalitions). The worth or payoff of a given coalition embedded in a partition is determined as follows: Coalitions determine their price offers jointly to maximize their expected overall (total) profit. A basic assumption of transferable-utility coalitional game theory applies here: we assume that the members of a coalition may freely redistribute their profits among themselves. We have to note that if we consider a different interpretation of coalition formation, and assume that different generators belong to different but cooperating companies, this is not necessary true in a realistic economic environment.

Based on the price offers the ISO determines the matching. The value of a coalition embedded in the partition $P$ is defined by the sum of the profits

\footnotetext{
4 Although it is possible that near the production capacity limits the validity of the decreasing marginal cost assumption is questionable, to keep the model as simple as possible, we restrict our analysis to the case where the linearly decreasing marginal cost assumption is valid. The model can be easily extended with more complex production characteristics.

5 The expected profit is the profit value which would be realized if all players outside the coalition would offer the same prices as in the previous step.
} 
corresponding to its members:

$$
V(C, P)=\sum_{j \in C} \Phi^{j}
$$

Since we allow the redistribution of profits among cooperating generators, and cooperation may affect agents not included in the coalition (externalities), the resulting game is defined in partition function form (Thrall and Lucas, 1963). Briefly, this means that the value of a certain coalition does depend on the partition in which it is embedded (in other words, the coalition structure of other players).

- The following market regulations are assumed:

- We assume that anti-cartel regulations exclude the formation of monopolistic coalitions.

Definition 3 We call a coalition monopolistic, if the presence of at least one member of the coalition is unavoidable in any matching which is stable and feasible.

Example Let us consider a 3 generator (nodes 1,2,3) 2 consumer (nodes $4,5)$ network. We summarize a matching with a two row matrix, in which a consumer (in the first row) is matched with the generator (in the second row) of the corresponding column. Eg. the matrix $\left(\begin{array}{ll}4 & 5 \\ 1 & 1\end{array}\right)$ means that both the consumers 4 and 5 are assigned to generator 1 . Let us furthermore assume that only the matchings $\left(\begin{array}{ll}4 & 5 \\ 1 & 1\end{array}\right),\left(\begin{array}{ll}4 & 5 \\ 2 & 2\end{array}\right)$, $\left(\begin{array}{ll}4 & 5 \\ 1 & 2\end{array}\right),\left(\begin{array}{ll}4 & 5 \\ 1 & 3\end{array}\right)$ and $\left(\begin{array}{ll}4 & 5 \\ 2 & 3\end{array}\right)$ are stable (in the sense of definition 2 ) and feasible (in the sense of definition 1). In this case none of the singleton coalitions or the coalitions $\{1,3\}$ or $\{2,3\}$ are monopolistic while the coalition $\{1,2\}$ is, because generator 1 or 2 appears in each possible (stable and feasible) matching.

The exclusion of monopolistic coalitions will result in the fact that despite any allowed cooperation, there will be at least two alternatives (considering coalitions) for any consumer, which implies a true competition. In other words we limit the validity of the model to those scenarios where the consumers have real alternatives It is reasonable to assume that if a monopolistic scenario arises, and the monopoly begins to vindicate its market power, the consumers will react with the decrease of the demand. Since as it has been discussed, our model assumes zero demand elasticity, and does not intend to analyze these cases.

- We assume zonal or general pricing. In the former case the generators may offer different prices for different consumers, while in the letter case every generator has to define one uniform price valid for all consumers.

- In the scenarios of symmetric information we assume that non-cooperating players have no information about each other's production characteristics 
( $a$ and $m$ ) but they are fully aware of the consumers' preference-setting principles, all offered prices in the previous step, and the ISO's matching algorithm. Asymmetric information assumptions are detailed in Section 3.3 .

- Regarding the iterative simulation of the model, generators are assumed to give price offers in each step, based on which consumers determine their preference over the generators. Based on these preferences the ISO determines a (stable and feasible) matching between consumers and generators and the corresponding transactions will be completed according to this matching.

- The generators publish their price offers simultaneously.

\subsection{Model formulation}

In this subsection, based on the assumptions enumerated in Section 2.2, we summarize the model parameters, elements, and variables. We assume that the coalitions are priori defined, fixed for the entire time of the simulations, and we analyze only partitions which do not include monopolistic coalitions.

Model Parameters:

- The $(N, E)$ graph of the network, including the admittance and maximal transmission capacity of the lines $E$, and the partitioning of the node set $N$ into generator and consumer nodes.

- The production characteristics of the generators $\left(a^{j}>0\right.$ and $m^{j}>0$ for generator $j$ ).

- The desired consumption values of the consumer nodes, and the generation capacities of the generator nodes.

Furthermore, the pricing algorithm of each coalition $\left(A^{C}\right)$ may vary. In game-theoretic terms, the players of the game are the generators and their strategy space is the set of prices offered for the consumers in each step.

\subsubsection{Consecutive offers and the PFF game of generators over the network}

We simulate the model in discrete time, where the time steps are denoted by the integer $t$. Since the offered prices (which are the model variables) change from time-step to time-step we introduce the argument $t$ as $p o_{k}^{j}(t)$. This implies that $P O$ is also a function of time, denoted by $P O(t)$.

The most important step in the simulation process is the determination of the generators' price offers, since these values will determine the consumers preferences. The algorithms based on which the generators determine their offered prices in each step are in general variable elements of the model.

\subsubsection{Simulation and optimization issues}

In the proposed framework every coalition $C$ tries to maximize its expected profit via its own algorithm $A^{C}$ while assuming that the other players of the 


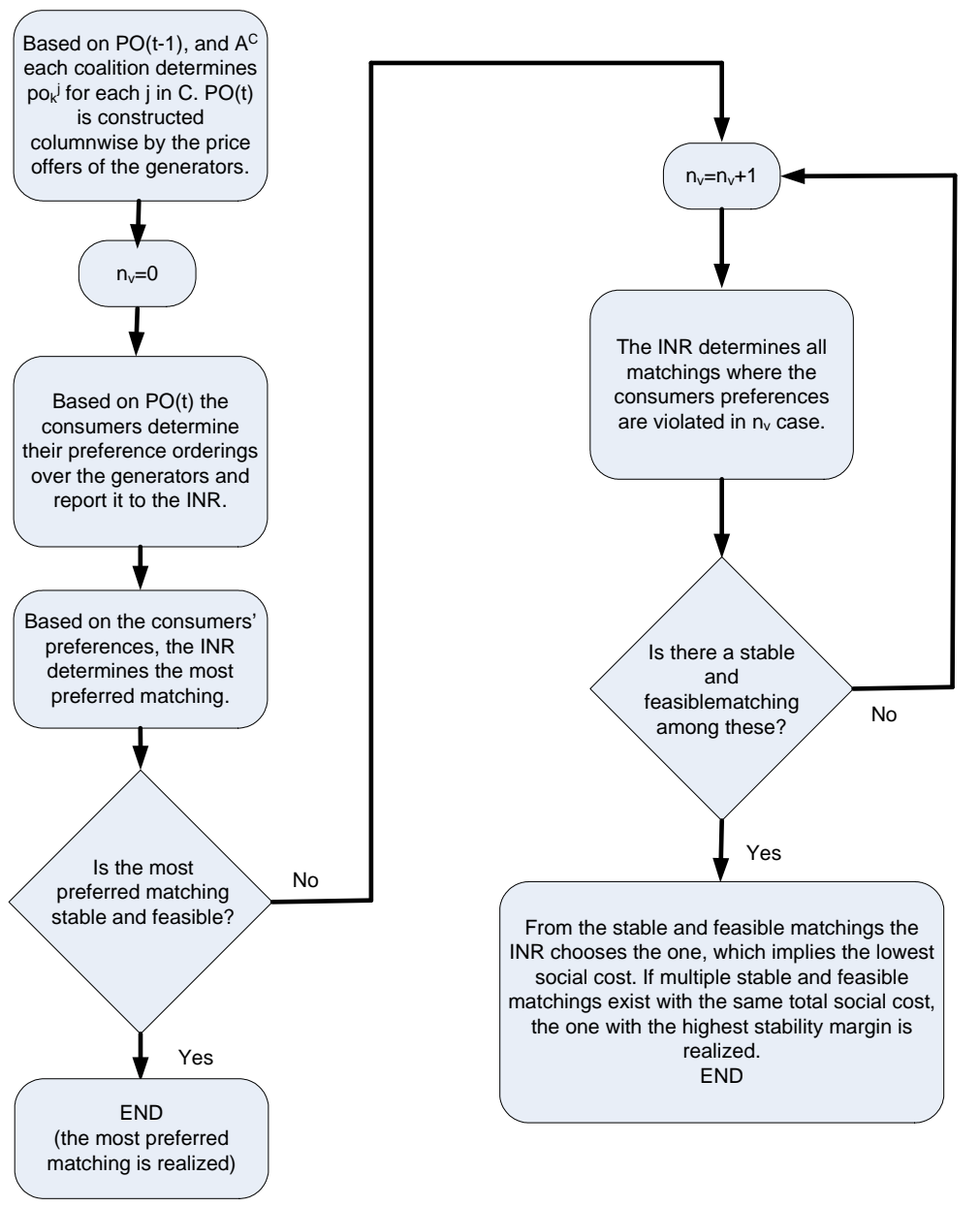

Fig. 1 The matching algorithm of the ISO.

game will offer the same prices as in the previous step. Formally this means that every coalition is trying to solve the optimization problem

$$
\max \sum_{j \in C} \Phi_{\text {exp }}^{j}\left(P O_{\text {exp }}(t)\right)=\mathcal{F}\left(\mu\left(\operatorname{Pref}\left(P O_{\text {exp }}(t)\right)\right)\right)
$$

where $\Phi_{\text {exp }}^{j}\left(P O_{\text {exp }}(t)\right)$ denotes the expected profit of player $j$ at time $t$. $P O_{\text {exp }}(t)$ denotes the matrix of expected price offers, which, for coalition $C$, apart from columns $j \in C$, is equal to $P O(t-1)$ (since all coalitions expect the outsider players not to change their price offers). Pref $\left(P O_{\exp }(t)\right)$ denotes the preference set up by the consumers according to $P O_{\text {exp }}(t)$ (every consumer defines an ordering of the generators according to increasing price offers). $\mu$ is defined by the matching algorithm of the ISO depicted in Fig. 1 , and $\mathcal{F}$ denotes the 
determination of the resulting profit from the matching as described in Section 2.2. The number of variables to be optimized depends on the coalition size and the market regulations (see later). If the actual coalitions has $k$ members and zonal pricing (different price offer for each consumer) is allowed, the number of variables is $k \cdot n_{c}$. If no zonal pricing is allowed, this number is simply $k$.

Taking into account that the objective function is discontinuous (the consumer's preferences change abruptly as the preference of consumers may change considering infinitesimally small price changes) and multiple local extrema may exist (expected profits may be increased either by raising prices, or via the possible reduction of prices which may lead to multiple consumers, higher production rate and so higher production efficiency) Therefore the offered price strongly depends on the capabilities of the algorithm used for the optimization of the expected profit function (see Appendix B).

This implies, that the proposed model is capable of comparing different optimization methods for the pricing problem, as in general it can be assumed that different players use different optimization methods, or they have different computing capacity.

Appendix B describes the comparison of various optimization methods for the pricing problem. Based on the results detailed there, and taking into account the desired feasibility of simulations, in this paper we assume that each player or coalition uses the particle swarm method for the optimization of the offered prices with a time limitation of 30 s in each step ${ }^{6}$.

Regarding scalability, the network-flow calculations are feasible even in the case of about hundred nodes (Fisher et al, 2008). The bottleneck is the two-stage pricing-optimization problem (1) of the coalitions. To determine its expected profit, a coalition in the proposed model has to be aware of matching algorithm of the ISO, which includes network-flow calculations and the minimization of the additional costs for the consumers whose preference is violated. Regarding zonal pricing, as the number of consumers, and so the number of price offers increase, the dimension of the search space corresponding to the optimization problem extends, which implies significant increase of the required computational time. If we constrain the calculation time of the particle swarm method, in such cases the standard deviation of the simulation results will be significantly larger. A possible approach may be to truncate the strategy space of the players: In the current model infinitely many price offers may be realized, since the strategy space (the offered price) for the players is continuous. If we allow eg. only 3 prices for each player (a low, a medium, and a high), and assume only 8 consumers, for 1 generator there are 6000+ possibilities of price offers, in each time step for each generator (and each evaluation includes network flow calculations). Furthermore in this case we get a mixed-integer problem. If we assume that one of the cooperating gener-

\footnotetext{
6 If we compare the difference between a time-constrained and not time constrained simulation results in the case of the proposed example, we can conclude that the variability is similar to the case when we compare eg. two time-unconstrained optimization results, due to the stochastic elements in the optimization process. This means that the 30 s constraint is acceptable in this case.
} 
ators is using the proposed explicit optimization while the other players use more simple rule-based methods or less computationally demanding optimization methods, the proposed framework may be applied easily with a tractable computational demand also in the case of larger problems. The basis of cooperation is the information in the proposed model - cooperating generators are aware of each others actual price offers. Such a scenario is however not capable of validating the hypotheses formulated later for zonal and general pricing in the case of larger networks, since the strategies of the players in this case are not symmetric as it will be in the proposed case. Fitting the problem for larger networks, and finding the optimization framework which can efficiently handle the non-continuous, many variable objective function may be a challenging task for the future.

Simulations detailed in the following were done using MATLAB. Since the particle swarm method used in the simulations has stochastic elements, the outcome of the pricing optimization may change from run to run. Accordingly, each simulation was done multiple times and a statistical average is presented and discussed. The simulation time-frame was determined as the shortest horizon in which the model produced significant results (see the discussion later).

\section{Equilibrium and convergence aspects}

As it has been mentioned equilibrium analysis is not included among the aims of this paper. Intuitively we can say that because of the various approaches via which a coalition may increase its profit (increase of offered prices on the one hand and decreasing them to divert customers from other generators), even the existence of the equilibrium for the defined problem is questionable. This intuition is supported by the fact that the offered prices in the simulations done never converged to a Nash equilibrium ${ }^{7}$ or limit cycle regardless of the simulation length, coalition structure, market regulations and asymmetricinformation assumptions. If the model converged to an equilibrium, or if the existence of such could be proved, it would be straightforward to define the coalition payoffs via the equilibrium profits.

Furthermore while in other models (see e.g. (Ruiz et al, 2012)) the nonzero level of demand elasticity (and e.g. piecewise constant price-demand curves and market clearing) may promote equilibrium, the assumed zero demand elasticity in the proposed model implies a different scenario.

\section{Results and discussion}

To demonstrate the game considered in Section 2.3 and some possibly arising phenomena we use the example network depicted in Fig. 2. The $Y_{s t}$ values denote the admittance values of the line connecting node $s$ and $t$. We suppose that the transmission-capacity constraint $\bar{q}_{s t}$, the maximum amount of

\footnotetext{
7 A Nash equilibrium in our model would mean a set of price offers that no coalition may improve its profit by unilaterally changing its price offer(s).
} 
energy which can be transmitted via the corresponding transmission line, is proportional to the admittance as $\bar{q}_{s t}=2.4 Y_{s t}{ }^{8}$.

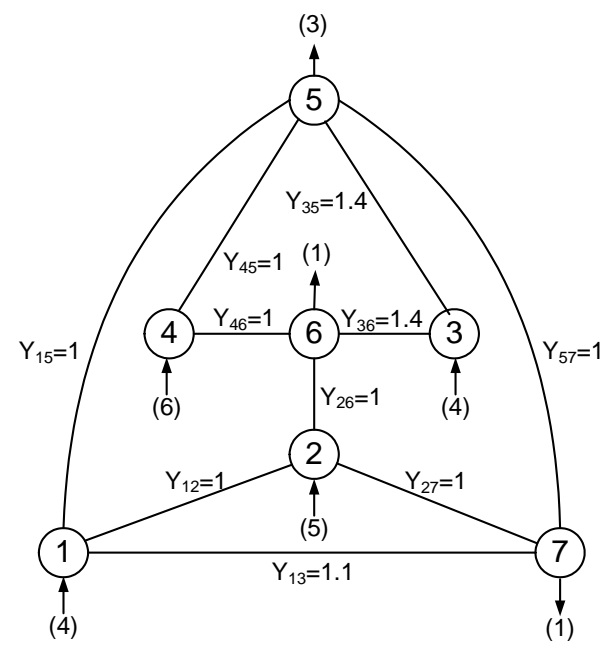

Fig. 2 Topology and parameters of network 1. The $Y_{s t}$ values denote the admittance values of the line connecting node $s$ and $t$ while $\bar{q}_{s t}$ denotes the transmission capacity constraint of line $s$ - $t$. The numbers next to the nodes indicate the available generation amounts and required consumption quantities denoted by inward and outward arrows respectively (denoted with $W$ in Appendix A.).

3.1 Cooperation may be necessary among generators to divert consumers from previous providers

In the following, first without the explicit analysis of profits and prices, we'll demonstrate how cooperating players may overcome limitations regarding networkstability during the extension of their client-set while diverting a consumer from a third generator. The further aim of this example is to demonstrate how the stability properties of the network may be a barrier for some matchings between generators and consumers.

Regarding the nodes in Fig. 2, 1, 2, 3 and 4 correspond to generators and 5, 6 and 7 correspond to consumers. At each node the desired consumption and maximal production values are indicated in parentheses. Nodes with arrows pointing from these values towards the nodes represent generators, while arrows pointing outward from the node indicate consumers. First let us identify

8 The value of 2.4 has been determined taking into account the following two considerations: First, the network shall be able to have a transmission capacity to fulfill the consumers needs (lower bound), and second, the transmission capacities have to impose real constraints on the stable matchings (upper bound). 
the monopolistic coalitions in the case of network 1 , since they are not allowed according to our assumptions. Since e.g. the following matchings

$$
\begin{aligned}
& \left(\begin{array}{lll}
5 & 6 & 7 \\
2 & 1 & 1
\end{array}\right) \quad\left(\begin{array}{lll}
5 & 6 & 7 \\
1 & 3 & 3
\end{array}\right) \quad\left(\begin{array}{lll}
5 & 6 & 7 \\
3 & 2 & 2
\end{array}\right) \quad\left(\begin{array}{lll}
5 & 6 & 7 \\
3 & 4 & 4
\end{array}\right) \\
& \left(\begin{array}{lll}
5 & 6 & 7 \\
2 & 4 & 4
\end{array}\right) \quad\left(\begin{array}{lll}
5 & 6 & 7 \\
1 & 4 & 4
\end{array}\right)
\end{aligned}
$$

are stable and feasible, we may conclude that all 2-player coalitions are nonmonopolistic. If we analyze further matchings, the calculations show that none of the generators is able to supply the consumers alone in a feasible and stable way. This implies that every coalition with at least 3 members is monopolistic.

Let us suppose the initial stable and feasible matching depicted in Fig. $3^{9}$

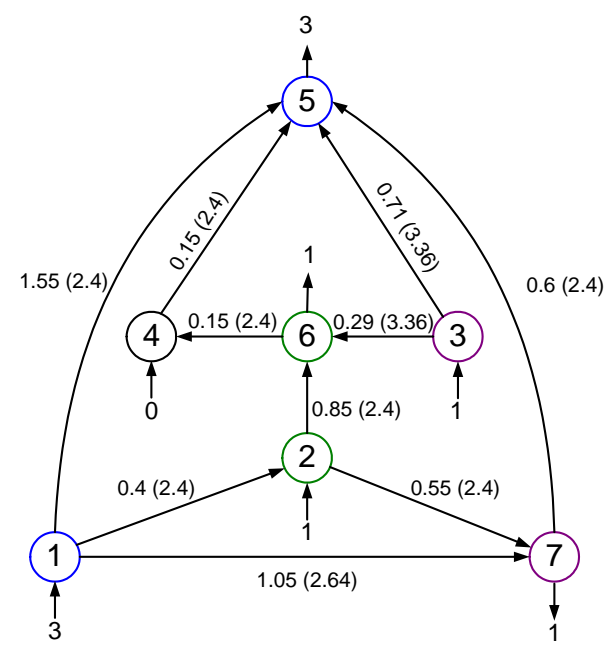

Fig. 3 Flows in the case of network 1, and the stable and feasible generator-consumer matching 5-1, 6-2, 7-3. Matchings are labeled with different colors. The numbers on the edges in parentheses denote the maximal flow value on the edge.

\subsubsection{Coalition structure $\{1\}\{2\},\{3\},\{4\}$}

Let us consider first the all-singleton partition $\{1\},\{2\},\{3\},\{4\}$, the initial stable and feasible matching depicted in Fig. 3 and the possible cooperation of generators 1 and 2. Let us assume that generator 1 offers a price for consumer 7 which is lower than the price offered by generator 3, its current supplier. In general, this can be a rational decision for generator 1, as the increase of

\footnotetext{
9 Results in Figures e.g. in Fig. 3 are only indicated in a 2 digit precision, which (due to rounding) may seem as inconsistencies: E.g. at node $51.55+0.15+0.71+0.6=3.01$ while the more accurate values are $1.5497+0.1471+0.7059+0.5973=3$.
} 
its load leads to more effective utilization of its capacities and this may imply even higher profits for him, even in the case of lower prices offered.

However, independent of the exact offered prices and potential profit change, although the capacity of generator 1 is sufficient to supply both consumers 5 and 7 , and no lines would be basically overloaded, this configuration is not allowed by the ISO, because of stability issues depicted in Fig. 4 .
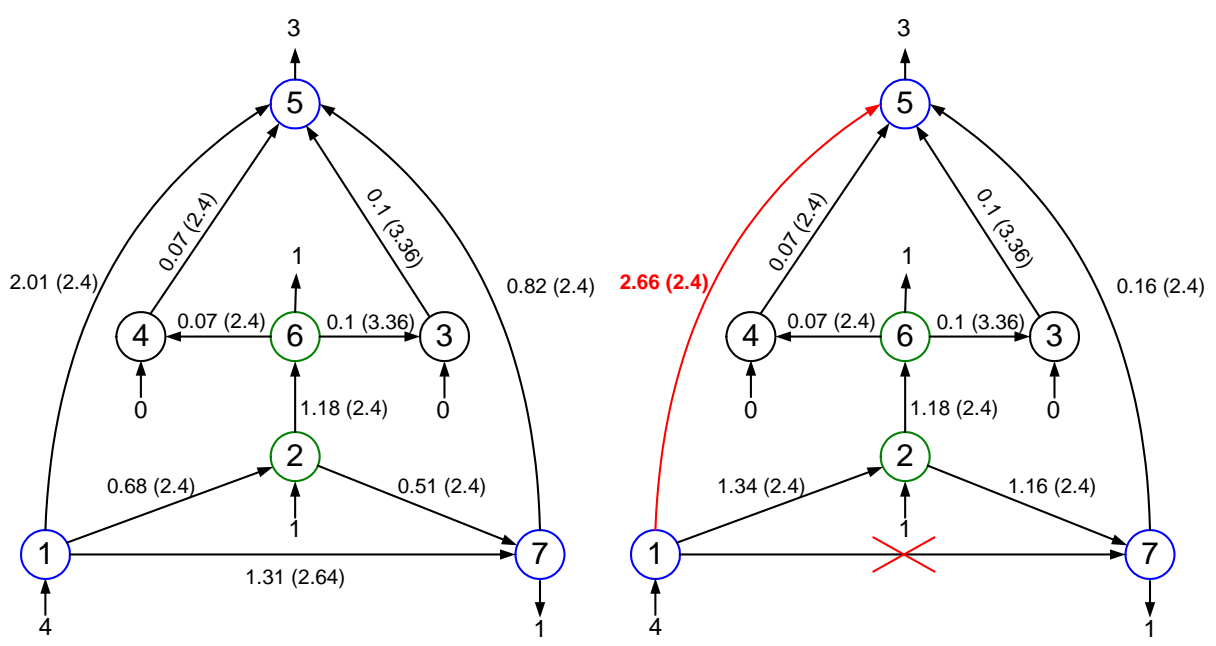

Fig. 4 If generator 1 would supply consumer 5 and 7 , the network would lose its stability (according to def. 2): The failure of line 1-7 would lead to the overload of line 1-5, so this configuration is not allowed by the ISO. However not included in the figure, further stability issues arise regarding this configuration: The failure of line 2-6 or 5-7 would also result in the overload of line 1-5.

As illustrated in Fig. 5 neither is Generator 2 able to supply consumer 7 in addition to its already established client, consumer 6 .

\subsubsection{Coalition structure $\{1,2\},\{3\},\{4\}$}

However, if Generators 1 and 2 are able to somehow arrange their prices in order to exchange their former consumers ( 5 and 6 ) between themselves, generator 1 is able to supply consumer 7 in addition to its former client 6 in a stable and feasible manner, as depicted in Fig 6.

It is important to note that, even in the case of cooperating generators, the consumers are still assigned to generators, not to coalitions of generators. The cooperating generators may however design their price offers jointly and thus influence the preference of consumers in a way to reach a beneficial resulting configuration. 

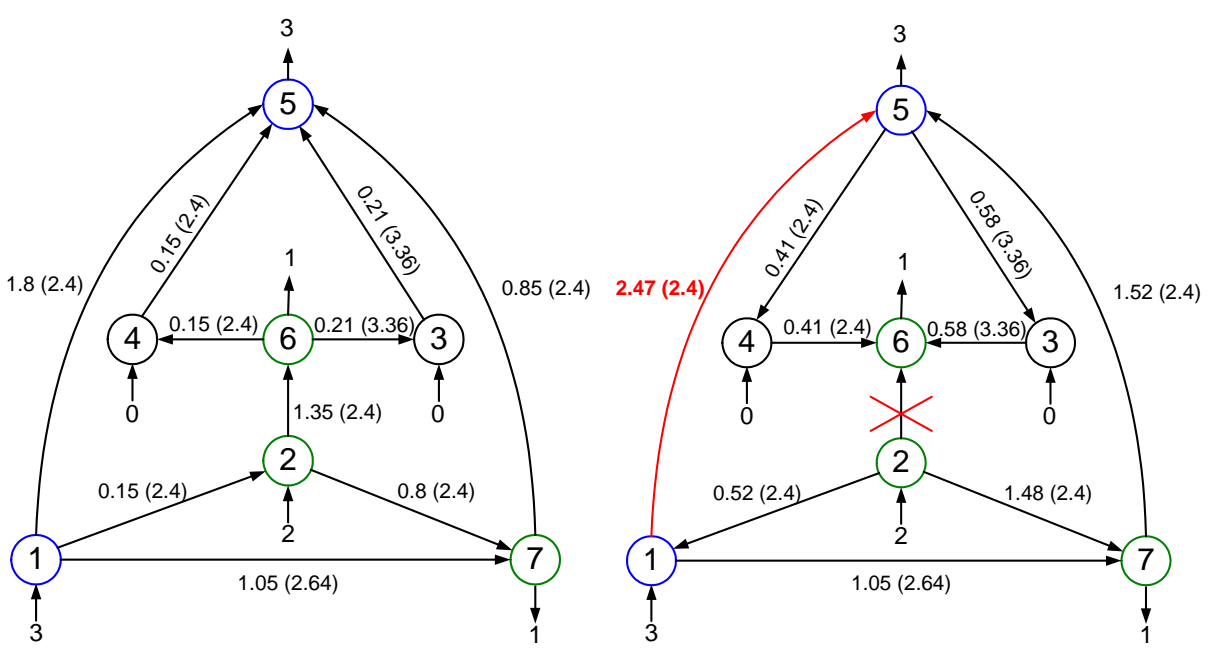

Fig. 5 If generator 2 would supply consumer 6 and 7 , the network would also lose its stability: The failure of line 2-6 would lead to the overload of line $1-5$, so this configuration is neither allowed by the ISO.

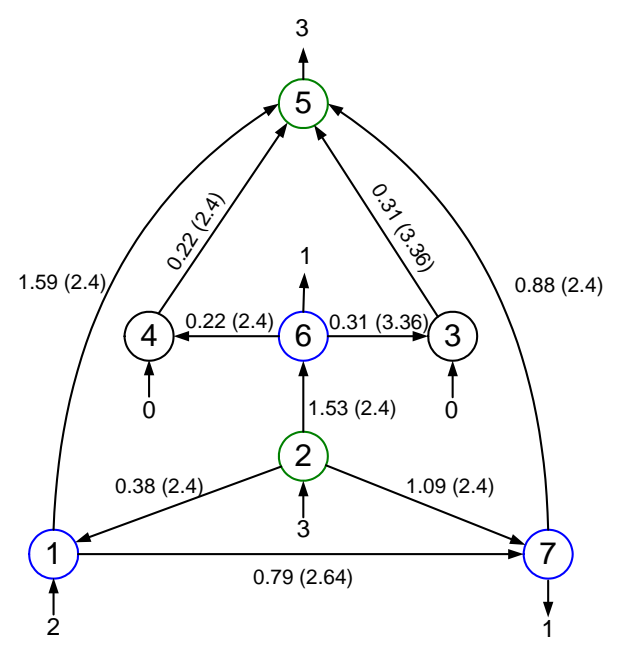

Fig. 6 Via cooperation, generators 1 and 2 are able to exchange their former consumers among themselves and divert consumer 7 from generator 3 .

3.2 Analysis of market regulations: Zonal versus global price offers

In the following we will analyze the PFF game defined in Section 2.3 over the given network (network 1) and player set. As mentioned, the value of a certain coalition embedded in a partition will be defined straightforwardly via the sum 
of its member's total profits. The total resulting profit of the players is derived via the evaluation of consecutive simulation steps as detailed in Section 2.3.1.

The first question we aim to analyze with our model is how the possibility of zonal pricing affects the profits of the generators and the total social cost. If zonal pricing is allowed each generator may offer its energy to each consumer on a different price.

\subsubsection{All-singleton coalitions, zonal pricing, example}

The scenario detailed next will serve as a reference for our future analysis to show some details of the evolution of profits. In this setup all players form singleton coalitions $\mathcal{Q}=\{\{1\},\{2\},\{3\},\{4\}\}$. Furthermore, in this reference case all generators are allowed to offer zone-dependent prices. In other words, they may offer their generated energy for sale for each individual consumer at a different price.

We assume the following production characteristic parameters: $a^{1}=0.65[\$]$, $a^{2}=0.36[\$], a^{3}=0.68[\$], a^{4}=0.7[\$], m^{1}=0.1[\$ / M W h], m^{2}=$ $0.07[\$ / M W h], m^{3}=0.08[\$ / M W h], m^{4}=0.04[\$ / M W h]$. Furthermore, let us assume the initial offered zonal prices are described by the following matrix

$$
P O(0)=\left(\begin{array}{cccc}
0.7 & 0.8 & 0.9 & 1 \\
0.85 & 0.75 & 0.95 & 1.05 \\
0.97 & 1.07 & 0.87 & 0.77
\end{array}\right)
$$

If we assume 10 iteration steps and calculate the price offers and the resulting matchings, the evolution of generator profits will be as depicted in Fig. 7. The number of iteration steps was determined to keep the computations feasible. The optimization takes 30s for each player or coalition and this has to be done in every partition and every step. As we will see these 10 steps are enough to show the phenomena we are interested in.

As we can see in Fig. 7 (a) in each step 2 or 3 generators supply the consumers with energy. At least one generator is lacking consumers in every step, which implies a profit of 0 . According to our simulation results the game does not tend to converge to a Nash equilibrium even if the number of iterations is increased by several orders of magnitude.

We can see that generator 4 whose $m$ parameter is the smallest amongst the 4 (he can increase his efficiency with the produced quantity the least) is usually able to reach the low profits. Furthermore, we have to note that in general profits may be also negative. This can be explained on the one hand by the assumption that if a generator offers a given price for a current timestep, in our model it has to fulfill all consumer demands towards him in that step, and on the other hand price offers of generators are based on the prices of competitors in the previous step (in other words, generators are rational in according to the information available about their competitors in the previous step and information about their coalitional partners at the actual step). If a generator was able to back off, the ISO would have to recalculate the matching in each such case. It is possible that a generator expects that it may supply 

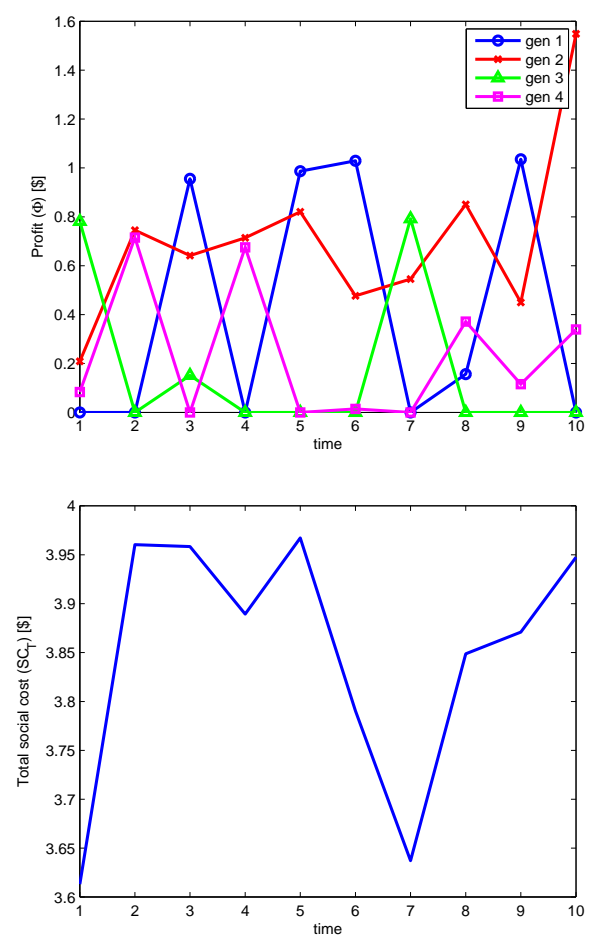

Fig. 7 (a) Evolution of generator profits in the reference case. Players do not cooperate, have no information about each other's production characteristic and are allowed to offer zonal prices for consumers. (b) The evolution of total social cost during the simulation.

multiple consumers and so utilize the more efficient part of its production characteristics, but the real scenario results only in a single customer for it. In this case, on the offered price, the income may not be even enough to cover the cost of energy production - this, as we see, is however not a typical scenario.

The evolution of the total social cost $S C_{T}$ during the 10 simulation steps is depicted in Fig. 7 (b).

\subsubsection{Zonal pricing}

If zonal pricing is allowed, the resulting partition function, single profits, and total social costs will be as summarized in Table 1.

Before the comparison of these results with the ones without zonal pricing available let us make a few observations. First, it can be observed that the results reflect the production characteristics. Generator 2, which has the most efficient production curve, reaches the highest profit in most of the cases, unlike generator 4, whose profit is usually among the lowest ones. Second, let us note that cooperation is almost always beneficial for the players. We 


\begin{tabular}{|c|c|c|c|c|c|c|}
\hline Partition & Values & $\Phi^{1}$ & $\Phi^{2}$ & $\Phi^{3}$ & $\Phi^{4}$ & $S C_{T}$ \\
\hline $1,2,3,4$ & $3.77,5.23,2.08,2.39$ & 3.77 & 5.23 & 2.08 & 2.39 & 37.21 \\
$\{1,2\}, 3,4$ & $9.23,4.34,2.45$ & 1.78 & 7.45 & 4.34 & 2.45 & 39.32 \\
$\{1,3\}, 2,4$ & $6.51,5.26,1.16$ & 4.56 & 5.26 & 1.95 & 1.16 & 36.07 \\
$\{1,4\}, 2,3$ & $\mathbf{5 . 7 6}, 5.83,2.88$ & 3.92 & 5.83 & 2.88 & 1.84 & 41.65 \\
$1,\{2,3\}, 4$ & $7.69,14.99,1.91$ & 7.69 & 13.56 & 1.43 & 1.91 & 40.27 \\
$1,\{2,4\}, 3$ & $3.84,10.96,2.8$ & 3.84 & 10.32 & 2.8 & 0.64 & 40.94 \\
$1,2,\{3,4\}$ & $2.33,7.84,8.04$ & 2.33 & 7.84 & 5.88 & 2.16 & 41.44 \\
$\{1,2\},\{3,4\}$ & $\mathbf{9 . 7 5}, 8.38$ & 3.11 & 6.64 & 5.42 & 2.96 & 43.84 \\
$\{1,3\},\{2,4\}$ & $6.97,7.38$ & 3.20 & 6.81 & 3.77 & 0.57 & 38.13 \\
$\{1,4\},\{2,3\}$ & $9.76,8.71$ & 5.92 & 5.83 & 2.88 & 3.84 & 41.65 \\
\hline
\end{tabular}

Table 1 Simulation results corresponding to zonal pricing. Bold typeface represents subadditive merging (when the resulting profit of a coalition is less than the sum of individual profits of its members - in other words strictly not superadditive). The results are the average of 10 simulations. The average standard deviation-values of the players profits were $\left[\begin{array}{llll}0.47 & 0.75 & 0.41 & 0.38\end{array}\right]$ respectively, while the average standard deviation of $S C_{T}$ was 1.01 .

use the game-theoretic concept of superadditivity for the analysis whether a cooperation is beneficial for its members or not. Formally if $\mathcal{Q}$ and $\mathcal{R}$ are partitions and $(\forall Q \in \mathcal{Q})(\exists R \in \mathcal{R})(Q \subseteq R)$, we say that $\mathcal{Q}$ is a refinement of $\mathcal{R}$. In this case, under superadditivity we mean that for the values of the coalitions (embedded in the corresponding partitions) the following inequality holds: $v\left(Q_{1}, \mathcal{Q}\right)+\ldots+v\left(Q_{k}, \mathcal{Q}\right) \leq v(R, \mathcal{R})$ where $R=\bigcup_{i=1}^{k} Q_{k}$. For example if we consider the emerging cooperation between player 1 and 2 , we have to analyze the superadditivity in two cases. First if players 3 and 4 are acting independently: in this case the total payoff of players 1 and 2 increases from 9 to 9.23 , and second if players 3 and 4 are forming a coalition, the payoff of players 1 and 2 practically decreases (10.17 vs. 9.75).

We can observe that cooperation is usually beneficial for the generators, this is not always the case: The network topology, production characteristics, and behavior of other players determine if players may increase their total payoff via cooperation or not ${ }^{10}$.

Furthermore, we may point out how important the assumption of transferable utility is. Although in general cooperation implies higher individual profits as well, this is not necessary. In the case eg. of the cooperation of players 2 and 4, players 1 and 3 may raise their resulting profit with cooperation from 6.64 to 6.97 but regarding the single generators while generator 3 benefits from the cooperation, generator 1 has to face a lower individual profit. In this case if generator 3 can not entirely transfer a part of its profit for the payoff to generator 1 entirely free, the cooperation may be reconsidered. Regarding externalities it can be easily seen (eg. the formation of $\{1,3\}$ from singletons) that coalition formation may imply both negative and positive externalities on the remaining players. In addition, we may compare the average total social cost in the cases where no multi-generator companies appear on the market (37.21), if one multi-generator company appears (39.95), and if 2

10 Of course cooperation implies that cooperating generators increase (or at least not decrease) their expected profit. However the realized profit may be significantly different. 
multi-generator companies appear (41.21). We can see that as expected, the presence of larger firms dampens the competition and results in higher prices for the consumers.

\subsubsection{General pricing}

If no zonal pricing is allowed, every generator offers its energy to every consumer at the same price. In this case, the initial conditions are taken as

$$
P O^{\text {init }}=\left(\begin{array}{llll}
0.85 & 0.8 & 0.9 & 1 \\
0.85 & 0.8 & 0.9 & 1 \\
0.85 & 0.8 & 0.9 & 1
\end{array}\right)
$$

In this case Table 2 summarizes the results.

\begin{tabular}{|c|c|c|c|c|c|c|}
\hline Partition & Values & $\Phi^{1}$ & $\Phi^{2}$ & $\Phi^{3}$ & $\Phi^{4}$ & $S C_{T}$ \\
\hline $1,2,3,4$ & $3.31,4.41,0.04,0.4$ & 3.31 & 4.41 & 0.04 & 0.4 & 29.4 \\
$\{1,2\}, 3,4$ & $8.54,0.76,0.29$ & 3.7 & 4.84 & 0.76 & 0.29 & 30.7 \\
$\{1,3\}, 2,4$ & $5.87,6.97,2.75$ & 3.11 & 6.97 & 2.75 & 0.79 & 35.78 \\
$\{1,4\}, 2,3$ & $7.32,8.11,0.77$ & 6.25 & 8.11 & 0.77 & 1.07 & 37.52 \\
$1,\{2,3\}, 4$ & $5.27,8.12,2.28$ & 5.27 & 6.64 & 1.48 & 2.28 & 37.31 \\
$1,\{2,4\}, 3$ & $3.44,5.25,0.52$ & 3.44 & 5.25 & 0.52 & 0 & 30.08 \\
$1,2,\{3,4\}$ & $2.74,3.28,1.26$ & 2.74 & 3.28 & 0.93 & 0.33 & 28.75 \\
$\{1,2\},\{3,4\}$ & $10.53,7.32$ & 0.84 & 9.69 & 4.9 & 2.42 & 43.76 \\
$\{1,3\},\{2,4\}$ & $11.84,9.36$ & 5.67 & 6.9 & 6.17 & 2.46 & 46.3 \\
$\{1,4\},\{2,3\}$ & $12.47,16.07$ & 6.24 & 12.2 & 1.80 & 2.89 & 47.53 \\
\hline
\end{tabular}

Table 2 Simulation results corresponding to general pricing. Bold typeface represents subadditive merging. The results are the average of 10 simulations. The average standard deviation values of the players profits were $\left[\begin{array}{llll}0.54 & 0.88 & 0.5 & 0.28\end{array}\right]$ respectively, while the average standard deviation of $S C_{T}$ was 1.36 .

Regarding superadditivity, in this case all of the coalitions meet the requirement.

The most important issue regarding the total social cost is that while the average prices in the case of none or one multi-generator company appears on the market $(29.4$ and $33.36=(30.7+35.78+37.52+37.31+30.08+$ $28.75) / 6$ ) respectively) is significantly lower than in the case of zonal pricing, the competition of two multi-generator companies result in very high average social cost (45.86) in the case of general pricing. We may state that our model simulations suggest the following: In the case of a highly competitive market, general pricing is beneficial for the consumers, while in the case of a market dominated by few multi-generator companies, zonal pricing may decrease the total social cost.

However we have to note that this hypothesis, yet plausible, is supported only by the proposed case study. The generalization of these results to larger networks requires more research on the subject. 


\subsection{Asymmetric information}

As we have seen in the examples before, generator 4, thanks to its disadvantageous production characteristics, was almost always able to reach only the lowest profits. In the following we will examine how much does it help if a generator is aware some of the other players offers. We suppose full competition (all-singleton coalitions) in this case.

In addition to the symmetric-information case, we will analyze 3 scenarios. Player 4 may be aware of one, two or all three price offers. If eg. player 4 has information of one other player's price offer, this player can be player 1,2 , or 3. Table 3 summarizes the average profits of the players and the average resulting total social cost in various cases of additional information of player 4.

\begin{tabular}{|c|c|c|c|c|c|}
\hline Information & $\Phi^{1}$ & $\Phi^{2}$ & $\Phi^{3}$ & $\Phi^{4}$ & $S C_{T}$ \\
\hline Symmetric & 3.77 & 5.23 & 2.08 & 2.39 & 37.21 \\
One additional offer & 3.79 & 8.84 & 4.37 & 2.63 & 43.26 \\
Two additional offers & 4.22 & 7.39 & 2.41 & 2.92 & 40.02 \\
Three additional offers & 2.98 & 7.2 & 3.71 & 3.42 & 47.29 \\
\hline
\end{tabular}

Table 3 Simulation results: Zonal Pricing, asymmetric information, full competition - additional information of player 4 . The results are the average of 10 simulations. The average standard-deviation values of the players profits were $\left[\begin{array}{llll}0.34 & 0.81 & 0.44 & 0.28\end{array}\right]$ respectively, while the average standard deviation of $S C_{T}$ was 1.29 .

We can see that although the additional information of one or two price offers increases the profit of player 4 , the real advantage is the case when it is aware of all other offers.

\begin{tabular}{|c|c|c|c|c|c|}
\hline Information & $\Phi^{1}$ & $\Phi^{2}$ & $\Phi^{3}$ & $\Phi^{4}$ & $S C_{T}$ \\
\hline Symmetric & 3.31 & 4.41 & 0.04 & 0.4 & 29.4 \\
One additional offer & 3.86 & 4.53 & 1.36 & 0.54 & 32.06 \\
Two additional offers & 3.91 & 3.75 & 1.14 & 0.27 & 31.37 \\
Three additional offers & 4.21 & 5.53 & 0.24 & 0.46 & 31.69 \\
\hline
\end{tabular}

Table 4 Simulation results: General Pricing, asymmetric information, full competition additional information of player 4 . The results are the average of 10 simulations. The average standard deviation values of the players profits were $\left[\begin{array}{llll}0.5 & 0.74 & 0.24 & 0.33\end{array}\right]$ respectively, while the average standard deviation of $S C_{T}$ was 1.12 .

Table 4 shows that under general pricing player 4 can not take significant advantage of the additional information as in the case of zonal pricing. The disadvantageous production characteristics and the low degree of freedom implied by the uniform prices can not be balanced by such additional information in this scenario. 


\section{Conclusions}

In this article a model framework is proposed, which is able to analyze the effect of cooperation, asymmetric information and market regulations on the profit of generator companies and on the cost of price preference consumers. In the demonstrated cases all players/coalitions were about to maximize their profit with numerical optimization based on the available information, however more simple strategies (eg. leader-follower) can also be assumed, which do not need this amount of computational effort. The particle-swarm optimization method applied during the simulations includes stochastic elements, which implies that the determined optimum may be not the same considering repetitive runs. Regarding the main conclusions of the simulations the variability of the results is acceptable, however according to this issue one primary further aim is to develop a more trusty and computationally efficient optimization algorithm for the pricing problem.

Furthermore, we have to note that even considering cooperation, the model assumes an essential competitive element in the market. The exclusion of monopolistic coalitions ensures that each consumer will have at least two alternatives for bargaining, regarding coalitions.

As the structure of power transmission networks may be subject to changes due to various reasons (eg. maintenance, installation of new lines), simulation studies similar to the proposed examples may help to determine how the changes in the network parameters affect the market power of existing/potential coalitions.

It is important to emphasize that the proposed model is far from being strictly realistic considering both the physical and the economic part. The transmission losses, which are currently not included in the model, would imply an excess in the total injected energy. Furthermore they would penalize generator-consumer pairs, which are far from each other and thus such matchings may result in completely different network-flow configurations and profits.

In addition in the case of real scenarios, energy suppliers dominantly use various generating units with diverse production characteristics to meet their consumers needs. While in the case of generating units with great inertia the rescheduling itself may imply significant costs, units of other types (eg. gas turbines) may be set up easily but can not be shut down and restarted economically. Of course one can not avoid the problem of renewable sources, which dominantly operate at very low production costs. They are subject to weather conditions and thus bring uncertainty to the system. One straightforward future direction for the development of the current model is to include these more complex production characteristics and probability-type variables in the system. We have to note that cooperative game theory already offers tools (Habis and Herings, 2011) to manage uncertainties. This approach has been used in the case of externalities and power transmission networks as well (Habis and Csercsik, 2014). 
Furthermore, our model also does not handle voltage-stability issues, which could be an additional factor to take into account while determining stable matchings of the network (Van Cutsem and Vournas, 1998).

As our model uses an iterative approach, one straightforward generalization assumption may be that the consumption needs are changing over time. This may affect the resulting profits multiple ways (smaller consumption amounts may be supplied by multiple providers, while maybe the competition boil down to the competition of two coalitions if the needed amount becomes greater).

Regarding economic aspects, also multiple future development directions can be considered. First of all, the model completely neglects the fee which is paid for the usage of the transmission network. The determination of transmission prices has been a subject of several studies (see eg. (Christie et al, 2000; Kirschen et al, 1997; Wijayatunga, 2003)). The proposed model can be quite easily extended to include transmission prices, and the effect of various transmission pricing strategies can be analyzed in the case of different scenarios.

At second, if we consider realistic energy providers, we have to assume that one company may hold multiple generation units, and the transaction takes place not between a consumer and a generator, but between the consumer and the power company. In this case the problem will be more complex. Since we may assume that the company tries to optimize its production cost, and that the quantity which has to be fed into the network by the company to meet its consumers needs may be generated at different nodes - it is likely that the generation configuration optimal for the company will not match the generation configuration optimal for network stability. In this case the power companies may be considered as players of the game.

We hope that the future extensions of the proposed model will be available to study the above problems also in the case of models of real power networks.

\section{A Appendix: DC Load flow models: a short summary}

In this appendix we introduce DC load flow models and point out some important properties of them. The notations and the mathematical formalism are based on Wu et al (1996) and Contreras (1997).

We consider a network with $n$ nodes (generators or consumers) and $m$ edges (transmission lines) among them. The network is given by the node-branch incidence matrix $A \in \mathbb{R}^{n \times m}$. We characterise generators and consumers by the quantity of actual $W \in \mathbb{R}_{+}^{n}$ and maximal $\bar{W} \in \mathbb{R}_{+}^{n}$ generated or, respectively, consumed power and transmission lines by the admittance matrix $Y$ (equivalently by the susceptance matrix $B$ ) and transmission capacity vector $\bar{Q} \in \mathbb{R}_{+}^{m}$. The power network can therefore be summarised as 5-tuple $(N, A, \bar{W}, Y, \bar{Q})$

The basic equations of the model are as follows.

The relation between the total inlet/outlet power and power flows (or nodal balances) can be described by

$$
A Q=W
$$

where $Q \in \mathbb{R}^{m}$ denotes the power flow vector, and $W \in \mathbb{R}^{n}$ is the power injection vector. 
On the other hand, if we assume that the nodes $i$ and $j$ are connected by a transmission line with admittance $Y_{i j}=Y_{j i}$, we have

$$
B \Theta=W
$$

where $W$ is the vector of the generated/consumed power at the nodes, $B \in \mathbb{R}^{n \times n}$ denotes the susceptance matrix whose elements are $B_{k l}=-Y_{k l}$ for the off-diagonal terms and

$$
B_{k k}=-\sum_{l \neq k} B_{k l}
$$

(the column sum of off-diagonals) for diagonal elements and $\Theta \in \mathbb{R}^{n}$ is vector of nodal voltage angles $\left(\theta_{i}\right)^{11}$. $\theta$ can be calculated as $\theta=B^{+} W$, where $B^{+}$is the Moore-Penrose pseudoinverse of $B$.

The admittance values determine the power flows via the voltage angles as

$$
q_{i j}=Y_{i j} \sin \left(\theta_{i}-\theta_{j}\right)
$$

Assuming that $\left(\theta_{i}-\theta_{j}\right)$ is small, $\sin (x)$ may be approximated with $x$. The flows can be determined as

$$
Q=B^{D} A^{T} \Theta
$$

where $B^{D}$ is a diagonal matrix with $B_{k k}^{D}=Y_{i j}$

This leads to the so called DC load flow model, in which the equations describing the power flow will me mathematically equivalent with the Kirchoff-equations describing current flow in DC voltage networks of resistors. DC load flow models exhibit the following uniqueness property.

Given power injections and power consumptions $W$ at each node, the phase angles $\theta_{i}$ are determined by solving a system of linear equations. From the phase angle differences, the line flows can be uniquely determined.

To point out some properties of the model let us consider the example with 3 nodes (2 generators, nodes 1 and 3 and 1 consumer, node 2) and 3 transmission lines. On Figure 8(a) we can see the ideal production/consumtion levels as well as the impedance and capacity levels. $q_{i j}$ shows the actual flows. In this example the optimal flow is not constrained by transmission capacities. We may calculate the voltage angles as $\theta=B^{+} W=\left[\begin{array}{ll}-2.42 & 2.74-\end{array}\right.$ $0.32]$ from which the flows can be calculated via equation 5 .

If we double the admittance and maximal transmission capacity between the nodes 2 and 3 (which can be regarded as the physical duplication of the power line, shown on Figure 8(b)), the line 1-3 will be overloaded, since the increased overall admittance of line 13-2 "draws the flow" onto that branch from 1-2. Of course a suitable reduction of production and consumption levels (moving away from the ideal values) leads to a feasible flow, not violating the line transmission constraints. If we reduce the power injections, the flows are reduced under the transmission capacities as in Fig. 8(c).

The constraint describing the maximum line power flows can be formulated as

$$
|Q|=\left|B^{D} A^{T} \Theta\right|=\left|B^{D} A^{T} B^{+} W\right|<\bar{Q}
$$

where $|\bar{Q}|$ is branch power flow limit vector (composed of the elements $\bar{q}_{i j}$ ), and $B^{D} \in \mathbb{R}^{m \times m}$ is a diagonal matrix with $B_{k k}^{D}=Y_{i j} . W$ is the variable power injection while $B^{D}, B^{+}, A^{T}$ and $\bar{Q}$ are derived from the parameters of the network as detailed above.

\section{A.1 $n-1$ line contingency}

$n-1$ line contingency means that the lines must not be overloaded in the case of a sudden line failure. A sudden failure means that the operators do not have time to reschedule the

11 In AC systems voltage is represented as a vector rotating on the complex plane. The angular speed of these vectors at the nodes is the same, but the actual angles may differ 


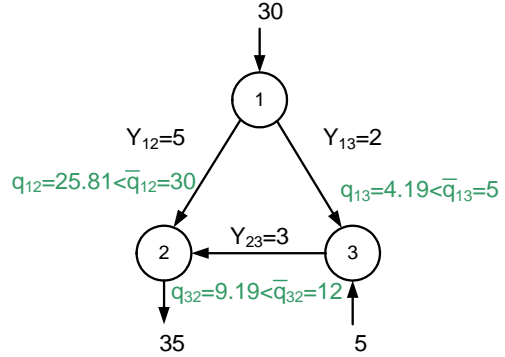

(a)

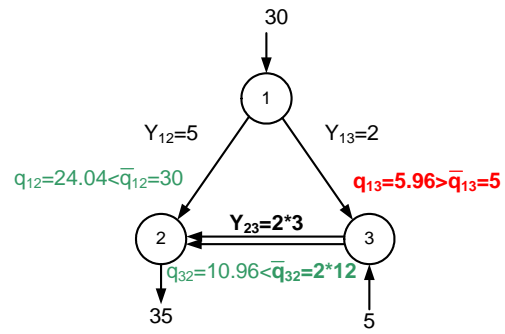

(b)

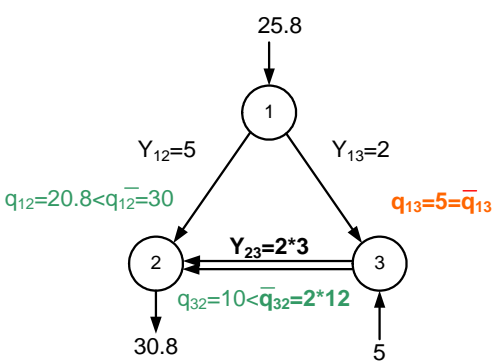

(c)

Fig. 8 Power flow on a simple 3-node network. The arrows pointing in and out of the nodes denote the power injections consumptions $(W)$. If we double the connection between 2 and 3, line 1-3 gets overloaded. $q_{i j}$ denotes the actual power flow on edge $i, j$ while $\bar{q}_{i j}$ denotes the maximum transmission capacity. $\Theta_{1}=\left[\begin{array}{lll}-2.42 & 2.74 & -0.32\end{array}\right]^{T}, \Theta_{2}=\left[\begin{array}{lll}-2.60 & 2.21 & 0.38\end{array}\right]^{T}$ and $\Theta_{3}=\left[\begin{array}{lll}-2.22 & 1.94 & 0.28\end{array}\right]^{T}$

generators (change the power inlets to the network). If a line fails, the matrices $A$ and $B$ change (originally nonzero elements become zero). If we denote the new matrices with $\hat{A}$ and $\hat{B}, n-1$ line contingency means that in addition to constraint (6), the following equation has to hold as well for all matrices implied by the possible single line failures.

$$
|Q|=\left|\hat{B}^{D} \hat{A}^{T} \Theta\right|=\left|\hat{B}^{D} \hat{A}^{T} \hat{B}^{+} W\right|<\bar{Q}
$$

Power injection vectors $(W)$ which result in the fulfillment of this property, are $n-1$ line contingency-safe. In this paper we call these configurations simply stable.

\section{B Appendix: Comparison of optimization methods for the pricing problem}

The most critical element of the proposed model is the determination of price offers. Since the expected profit-optimization problem (see. Eq. 1) is not trivial because of the discontinuities of the objective function and multiple local extrema. Furthermore one or more from these local extrema usually lie in the neighborhood of the discontinuities. The reason for this is that a generator $g$ may increase its expected profit with the increase of the price offered to one customer, until the offered price becomes equal or greater compared to the price offered by one of its competitors, in which case (if the corresponding matching is stable and feasible) the consumer will be diverted from the generator $g$, and the expected profit corresponding to this transaction falls to 0 . On the other hand, as mentioned earlier, with the lowering of its 
offered prices generator $g$ may divert customers of competitors and enhance the efficiency of its production. The existence of local multiple extrema calls for global optimization methods, however we include a local approach as well for reference.

In this appendix we compare several optimization methods for this pricing problem. The algorithms analyzed are the following.

- Simulated annealing (van Laarhoven and Aarts, 2008) (SA), as implemented in the MATLAB function simulannealbnd.

- NOMAD Nonlinear optimization with the MADS algorithm (Le Digabel, 2011).

- Particle swarm (PS) pattern search method (Vaz and Vicente, 2007).

- NLOPT (Johnson, 2010) (with the solver GN_DIRECT).

- IPOPT Interior Point Optimizer (Wächter and Biegler, 2006) (local optimization).

The later 4 functions were obtained by the OPTI toolbox (Currie and Wilson, 2012).

The comparison was performed as follows. All of the above methods were used to solve the pricing optimization problem in a simulation of 25 steps with 4 players. In each step for each player, each method was used to perform the optimization and the resulting values of the objective function were compared (since computationally minimization is performed but the objective is to maximize the expected profit, the $-\Phi_{\text {exp }}^{j}$ was used). Considering that the price optimization process during the simulations has to be carried out in each time step for each coalition, the maximal computation time of each algorithm was limited to 30s. In each case the results of different methods were compared to the minimum value obtained by the actually most effective algorithm. The results are depicted in Fig. 9.

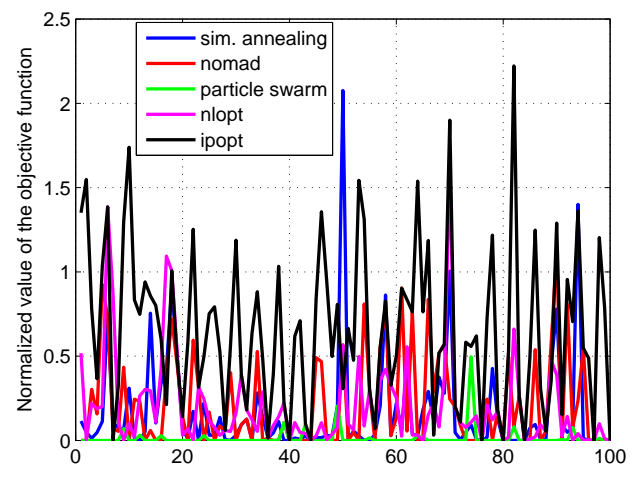

Fig. 9 Performance of the various optimization approaches in the case of the pricing problem.

At first glance it can be seen in Fig. 9 that, as expected, the local method IPOPT is less effective in most of the cases compared to the global approaches. We may use the integral of the curves depicted in Fig. 9 to quantitatively characterize the efficiency of the methods. We denote the accumulated difference compared to the best solution with $F_{A}$.

\begin{tabular}{|c|c|c|c|c|c|}
\hline Method & SA & NOMAD & PS & NLOPT & IPOPT \\
\hline$F_{A}$ & 16.74 & 21.32 & 1.30 & 20.32 & 66.13 \\
\hline
\end{tabular}

Table 5 Accumulated performance of optimization approaches in the case of the pricing problem. 
The results in table 5 show that in most of the cases the PS method provides the best values, and it shows the best performance among the analyzed methods.

Considering the 30s computation time limitation of the optimization, a further preliminary experiment was performed to estimate the convergence properties of the PS algorithm. A simulation of 10 steps and 4 players was performed. After every optimization procedure performed by the PS, the resulting values were used as initial condition for the local optimization method fminsearch, which uses the gradient-free Nelder-Mead simplex method (Olsson and Nelson, 1975) to validate the local minimality of the solution points. Analyzing these 40 realistic cases, the result was that the simplex method with maximum 250 iterations (meaning about one minute additional computational time in each case) was not able to enhance the value of the objective function significantly (by more than $0.01 \%$ ) in half of the cases. Moreover, the increase was below $5 \%$ in $85 \%$ of the cases, with the greatest improvement brought by the simplex being about $15 \%$ (in one case). Based on these preliminary simulations it can be concluded that the PS with the above time limitation means an acceptable trade-off for the pricing optimization problem.

\section{References}

Arnold T, Schwalbe U (2002) Dynamic coalition formation and the core. Journal of Economic Behaviour \& Organization 49(3):363-380, DOI 10.1016/S0167-2681(02)00015-X, URL http://dx.doi.org/10.1016/S0167-2681(02)00015-X

Bai X, Shahidehpour S, Ramesh V (1997) Transmission analysis by nash game method. IEEE Transactions on Power Systems 12:1046-1052

Bakirtzis AG, Ziogos NP, Tellidou AC, Bakirtzis GA (2007) Electricity producer offering strategies in day-ahead energy market with step-wise offers. Power Systems, IEEE Transactions on 22(4):1804-1818

Berndt ER, Wood DO (1975) Technology, prices, and the derived demand for energy. The review of Economics and Statistics pp 259-268

Bernstein MA, Griffin JM, Infrastructure S (2006) Regional differences in the price-elasticity of demand for energy. National Renewable Energy Laboratory

Bolle F (1992) Supply function equilibria and the danger of tacit collusion: the case of spot markets for electricity. Energy Economics 14(2):94-102

Cardell JB, Hitt CC, Hogan WW (1997) Market power and strategic interaction in electricity networks. Resource and Energy Economics 19(1-2):109-137, DOI 10.1016/S09287655(97)00006-7, URL http://dx.doi.org/10.1016/S0928-7655(97)00006-7

Chen Y, Hobbs B, Leyffer S, Munson TS (2006) Leader-follower equilibria for electric power and $\mathrm{NO}_{x}$ allowances markets. Computational Management Science 3:307-330

Cheng CP, Liu CW, Liu CC (2000) Unit commitment by lagrangian relaxation and genetic algorithms. Power Systems, IEEE Transactions on 15(2):707-714

Christie R, Wollenberg B, Wangensteen I (2000) Transmission management in the deregulated environment. IEEE Transactions on Power Systems 88:170-195

Conejo AJ, Aguado JA (1998) Multi-area coordinated decentralized dc optimal power flow. Power Systems, IEEE Transactions on 13(4):1272-1278

Contreras J (1997) A cooperative game theory approach to transmission planning in power systems. PhD thesis, University of California, Berkeley

Contreras J, Wu F (1999) Coalition formation in transmission expansion planning. IEEE Transactions on Power Systems 14:1144-1152

Contreras J, Wu F (2000) A kernel-oriented algorithm for transmission expansion planning. IEEE Transactions on Power Systems 15:1434-1440

Contreras J, Gross G, Arroyo JM, Muñoz JI (2009) An incentive-based mechanism for transmission asset investment. Decis Support Syst 47:22-31, DOI 10.1016/j.dss.2008.12.005, URL http://dl.acm.org/citation.cfm?id=1519538.1519674

Csercsik D, Kóczy LÁ (2011) Externalities in the games over electrical power transmission networks. Discussion Paper 2011.25, Centre for Economic Studies, Budapest, URL http://econ.core.hu/file/download/mtdp/MTDP1125.pdf 
Currie J, Wilson DI (2012) Opti: Lowering the barrier between open source optimizers and the industrial matlab user. Foundations of Computer-Aided Process Operations, Savannah, Georgia, USA pp 8-11

Ding F, Fuller J (2005) Nodal, uniform, or zonal pricing: distribution of economic surplus. Power Systems, IEEE Transactions on 20(2):875 - 882, DOI 10.1109/TPWRS.2005.846042

Ehrenmann A (2004) Equilibrium problems with equilibrium constraints and their application to electricity markets. Fitzwilliam College, Fitzwilliam 154

Evans F, Zolezzi J, Rudnick H (2003) Cost assignment model for electrical transmission system expansion: an approach through the kernel theory. IEEE Transactions on Power Systems 18:625-632

Fisher EB, O'Neill RP, Ferris MC (2008) Optimal transmission switching. Power Systems, IEEE Transactions on 23(3):1346-1355

Fuller J (2005) Relations among prices at adjacent nodes in an electric transmission network. Networks and Spatial Economics 5(3):279-292, DOI 10.1007/s11067-005-3036-0, URL http://dx.doi.org/10.1007/s11067-005-3036-0

Gabriel S, Siddiqui S, Conejo A, Ruiz C (2013) Solving discretely-constrained nashcournot games with an application to power markets. Networks and Spatial Economics 13(3):307326, DOI 10.1007/s11067-012-9182-2, URL http://dx.doi.org/10.1007/s11067-012-9182-2

Gately D (1974) Sharing the gains from regional cooperation: A game theoretic application to planning investment in electric power. International Economic Review 15:195-208

Gilbert R, Neuhoff K, Newbery D (2004) Allocating Transmission to Mitigate Market Power in Electricity Networks. RAND Journal of Economics 35(4):691--709, URL http://www.jstor.org/pss/1593768

Habis H, Csercsik D (2014) Cooperation with externalities and uncertainty. Networks and Spatial Economics pp 1-16, DOI 10.1007/s11067-014-9265-3, URL http://dx.doi.org/10.1007/s11067-014-9265-3

Habis H, Herings PJJ (2011) Transferable utility games with uncertainty. Journal of Economic Theory 146(5):2126-2139, URL http://ideas.repec.org/a/eee/jetheo/v146y2011i5p2126-2139.html

Harrington J, Hobbs BF, Pang JS, Liu A, Roch G (2005) Collusive game solutions via optimization. Mathematical programming 104(2-3):407-435

Hedman KW, O'Neill RP, Fisher EB, Oren SS (2008) Optimal transmission switchingsensitivity analysis and extensions. Power Systems, IEEE Transactions on 23(3):1469-1479

Hedman KW, O'Neill RP, Fisher EB, Oren SS (2009) Optimal transmission switching with contingency analysis. Power Systems, IEEE Transactions on 24(3):1577-1586

Hedman KW, Ferris MC, O’Neill RP, Fisher EB, Oren SS (2010) Co-optimization of generation unit commitment and transmission switching with n-1 reliability. Power Systems, IEEE Transactions on 25(2):1052-1063

Hingorani NG (1993) Flexible ac transmission. spectrum, IEEE 30(4):40-45

Hingorani NG, Gyugyi L, El-Hawary M (2000) Understanding FACTS: concepts and technology of flexible AC transmission systems, vol 1. IEEE press New York

Hobbs B, Kelly K (1992) Using game theory to analyze electric transmission pricing policies in the united states. European Journal of Operational Research 56:154-171

Johnson SG (2010) The nlopt nonlinear-optimization package

Kirschen D, Strbac G (2004) Fundamentals of Power System Economics. John Wiley \& Sons, Ltd, Chichester, UK, DOI 10.1002/0470020598, URL http://doi.wiley.com/10.1002/0470020598

Kirschen D, Allan R, Strbac G (1997) Contributions of individual generators to loads and flows. IEEE Transactions on Power Systems 12:52-60

Kleindorfer P, Wu DJ, Fernando C (2001) Strategic gaming in electric power markets. European Journal of Operational Research 130:156-168

Le Digabel S (2011) Algorithm 909: Nomad: Nonlinear optimization with the mads algorithm. ACM Transactions on Mathematical Software (TOMS) 37(4):44

Leuthold F, Weigt H, von Hirschhausen C (2012) A large-scale spatial optimization model of the european electricity market. Networks and Spatial Economics 12(1):75-107, DOI 10.1007/s11067-010-9148-1, URL http://dx.doi.org/10.1007/s11067-010-9148-1 
Liu A, Hobbs B (2013) Tacit collusion games in pool-based electricity markets under transmission constraints. Mathematical Programming 140(2):351-379, DOI 10.1007/s10107013-0693-5, URL http://dx.doi.org/10.1007/s10107-013-0693-5

Metzler C, Hobbs B, Pang JS (2003) Nash-Cournot equilibria in power markets on a linearized dc network with arbitrage: Formulations and properties. Networks and Spatial Economics 3(2):123-150, DOI 10.1023/A:1023907818360

Neuhoff K, Barquin J, Boots M, Ehrenmann A, Hobbs B, Rijkers F, Vázquez M (2005) Network-constrained Cournot models of liberalized electricity markets: the devil is in the details. Energy Economics 27:495-525

Oggioni G, Smeers Y, Allevi E, Schaible S (2012) A generalized Nash equilibrium model of market coupling in the european power system. Networks and Spatial Economics 12(4):503-560, DOI 10.1007/s11067-011-9166-7, URL http://dx.doi.org/10.1007/s11067011-9166-7

Olsson DM, Nelson LS (1975) The nelder-mead simplex procedure for function minimization. Technometrics 17(1):45-51

Orths A, Schmidtt A, Styczynski Z, Verstege J (2001) Multi-criteria optimization methods for planning and operation of electrical energy systems. Electrical Engineering 83:251-258

ONeill RP, Hedman KW, Krall EA, Papavasiliou A, Oren SS (2010) Economic analysis of the $\mathrm{n}-1$ reliable unit commitment and transmission switching problem using duality concepts. Energy Systems 1(2):165-195

Ruiz C, Conejo AJ, Smeers Y (2012) Equilibria in an oligopolistic electricity pool with stepwise offer curves. Power Systems, IEEE Transactions on 27(2):752-761

Sauma EE, Oren SS (2007) Economic criteria for planning transmission investment in restructured electricity markets. Power Systems, IEEE Transactions on 22(4):1394-1405

Sheble GB, Fahd GN (1994) Unit commitment literature synopsis. Power Systems, IEEE Transactions on 9(1):128-135

Singh H, Hao S, Papalexopoulos A (1998) Transmission congestion management in competitive electricity markets. Power Systems, IEEE Transactions on 13(2):672 -680, DOI $10.1109 / 59.667399$

Song YH, Johns AT (1999) Flexible ac transmission systems (FACTS), vol 30. IET

Thrall RM, Lucas WF (1963) n-person games in partition function form. Naval Research Logistics Quarterly 10(4):281-298

De la Torre S, Conejo A, Contreras J (2003) Simulating oligopolistic pool-based electricity markets: A multiperiod approach. Power Systems, IEEE Transactions on 18(4):1547-1555

De la Torre S, Contreras J, Conejo AJ (2004) Finding multiperiod nash equilibria in poolbased electricity markets. Power Systems, IEEE Transactions on 19(1):643-651

Tseng CL, Oren SS, Cheng CS, Li Ca, Svoboda AJ, Johnson RB (1999) A transmissionconstrained unit commitment method in power system scheduling. Decision Support Systems 24(3):297-310

Van Cutsem T, Vournas C (1998) Voltage Stability of Electric Power Systems. Kluwer Academic Publishers

van Laarhoven P, Aarts E (2008) Simulated Annealing: Theory and Applications. Springer

Vaz AIF, Vicente LN (2007) A particle swarm pattern search method for bound constrained global optimization. Journal of Global Optimization 39(2):197-219

Wächter A, Biegler LT (2006) On the implementation of an interior-point filter line-search algorithm for large-scale nonlinear programming. Mathematical programming 106(1):2557

Wijayatunga P (2003) Optimal transmission pricing with generation uncertainty and transmission losses. IEEE Transactions on Power Systems 150:73-77

Wood AJ, Wollenberg BF (2012) Power generation, operation, and control. John Wiley \& Sons

Wu F, Varaiya P, Spiller P, Oren S (1996) Folk theorems on transmission access: Proofs and counterexamples. Journal of Regulatory Economics 10(1):5-23, DOI 10.1007/BF00133356, URL http://dx.doi.org/10.1007/BF00133356

Yao J, Oren SS, Adler I (2004) Computing cournot equilibria in two settlement electricity markets with transmission constraint. In: System Sciences, 2004. Proceedings of the 37th Annual Hawaii International Conference on, IEEE, pp 9-pp 
Zhuang F, Galiana F (1990) Unit commitment by simulated annealing. Power Systems, IEEE Transactions on 5(1):311-318 